Western University

Scholarship@Western

Department of Economics Research Reports

Economics Working Papers Archive

1981

\title{
The Impact of Imperfectly Categorizing Risks on Income Inequality and Social Welfare
}

Michael Hoy

Follow this and additional works at: https://ir.lib.uwo.ca/economicsresrpt

Part of the Economics Commons

Citation of this paper:

Hoy, Michael. "The Impact of Imperfectly Categorizing Risks on Income Inequality and Social Welfare." Department of Economics Research Reports, 8111. London, ON: Department of Economics, University of Western Ontario (1981). 
ISSN: $\quad 0318-725 \mathrm{X}$

ISBN : $0-7714-0283-\mathrm{X}$

\section{RESEARCH REPORT 8111}

THE IMPACT OF IMPERFECTLY CATEGORIZING

RISKS ON INCOME INEQUALITY AND SOCIAL WELFARE

by

Michael Hoy

Ju1y 1981

Department of Economics Library

JUL 291981

Univensity of Westers Ontario 


\section{Abstract}

The use of imperfect information to categorize risks is a pervasive phenomenon in the insurance industry. Although this activity reduces the "average" extent of vertical price discrimination it is shown that both high and low risk types may prefer, ex ante to knowing the category to which they will be assigned, that such information be suppressed. Furthermore, for the Lorenz criterion it is shown that under certain restrictive demand conditions, the imperfect categorization of risks leads to an increase in inequality. The paper employs the Wilson E2 equilibrium concept. 


\section{INTRODUCTION}

The use of imperfect information to categorize risks is a pervasive phenomenon in insurance markets. When consumers know the risk class to which they belong insurance firms attempt to acquire this information in order to avoid the problem of adverse selection. The information which firms can "economically" employ is often imperfect and, therefore, leads to the imperfect categorization of risks. In a previous paper (see Hoy [1981]) it was shown that for the Wilson E2 equilibrium, ${ }^{1}$ it may be the case that this activity will lead to a Pareto-type improvement in welfare. However, this possibility requires the special assumption that the initial equilibrium (i.e., before categorization) not be a pooling one. ${ }^{2}$

In this paper the results of categorization are investigated for the case with all the equilibria, both before and after categorization, being of the pooling variety. It is demonstrated that if the coinsurance rate does not change after categorization (i.e., for either risk category) then an increase in income inequality is the result of imperfect categorization; that is, the Lorenz curve for the distribution of wealth displays an unambiguous increase in inequality after categorization.

It is often suggested that the presence of high risk types, when they cannot be differentiated from low risk types, imposes an externality on the low risk types. The reason is that the presence of unidentifiable high risk types restricts the ability of firms to offer full insurance to low risk types at their actuarially fair rate. If firms were to offer such a contract, high risk types would also purchase it and the firm would incur expected losses. One alternative for firms is to offer a single contract at the pooled fair odds 
price. In this case low risk types are faced with a price of insurance which is greater than the actuarial cost of providing insurance to them.

The use of imperfect information to categorize risks reduces the proportion of high risk types in the low risk category and increases this proportion in the high risk category. Since low risk types are more likely to be assigned to the low risk category than are high risk types it follows that the average price of insurance faced by each risk type after categorization more accurately reflects the expected cost of being provided with the coverage. In particular, low risk types face a lower average price for insurance after categorization. Therefore, one might argue that, despite any adverse effects on the distribution of wealth, imperfect categorization schemes are fair and, when possible, should be implemented since they reduce the "average extent" of the adverse price discrimination ${ }^{3}$ imposed on low risk types by the presence of high risk types. However, it is shown in this paper that, ex ante to knowing the risk category to which one will be assigned, low risk types may prefer that the information be suppressed and that the categorization scheme not be implemented. This result follows even though the expected price of insurance, for low risk types, which results from a prospective categorization scheme is less than the price with no categorization.

The model used in this paper is described in section II. In Section III it is shown that if one assumes that the coinsurance rate doesn't change after categorization (for either category) then an unambiguous increase in income inequality is the result of imperfectly categorizing risks. This result is shown to be robust in the (weak) sense that small perturbations from the assumption of a constant coinsurance rate will not change the result. Furthermore, abandoning this assumption altogether does not naturally lead to an 
improvement in the equality of the distribution of wealth after categorization (i.e., relative to the case with a constant coinsurance rate). In Section IV an example is presented which demonstrates that, ex ante to the revelation of which individuals are assigned to which risk category, both high and low risk types may prefer that a categorization scheme not be implemented. This result follows even though low risk types are faced with a higher probability of being assigned to the low risk category than are high risk types. A summary is provided in Section $\mathrm{V}$.

\section{THE MODEL}

There are two risk types, $i=1,2$, who incur loss $d$ with probability $p_{i}$. The high risk types are denoted by the subscript $i=1$ while the low risk types are denoted by the subscript $i=2$ (i.e., $p_{1}>p_{2}$ ). It is assumed that high and low risk types are risk averse and have identical utility functions and equal initial wealth.

It is assumed that there are no administrative costs associated with the provision of insurance. Firms are risk neutral and behave according to the Wilson E2 equilibrium concept. According to this equilibrium concept firms possess sufficient foresight so that they do not offer contracts which, even if they initially earn positive expected profits, will ultimately earn negative expected profits after other firms react. 4

The result of the above assumptions is that the equilibrium, if a pooling one, will be that point which corresponds to the highest indifference curve for low risk types at the pooled fair price. This is demonstrated in 
Figure I below. E corresponds to the initial endowment (i.e., with no insurance). EL refers to the actuarially fair odds line for low risk types and $\mathrm{EH}$ refers to the actuarially fair odds line for high risk types. The pooled fair odds line is EF. Its position depends on the proportion of high and low risk types in the population. The higher is the proportion of high risk types the closer will EF be to EH while the smaller is the proportion of high risk types the closer will EF be to EL. The Wilson E2 equilibrium is represented by $\alpha$. Since the pooled price is greater than the actuarially fair price for low risk types, less than full coverage will be "chosen."

The impact of employing imperfect information relating to risk class membership is demonstrated in Figure $I$. This information allows firms to (costlessly) assign individuals to one of two categories. The categories are such that the high risk category contains a larger proportion of high risk types (and the low risk category contains a smaller proportion of high risk types) than does the aggregate population. The result is that $\mathrm{EF}_{1}$, which corresponds to the (pooled) actuarially fair odds line for the high risk. category lies to the left of EF but, since the information is imperfect, lies to the right of EH. Similarly, $\mathrm{EF}_{2}$ lies between EF and EL.

The Wilson E2 pooling equilibrium for the high risk category is $\alpha_{1}$ while it is $\alpha_{2}$ for the low risk category. It was shown in Hoy [1981] and can be seen in Figure I that individuals assigned to the high risk category are made worse off by categorization while those assigned to the low risk category are made better off. 
Figure I

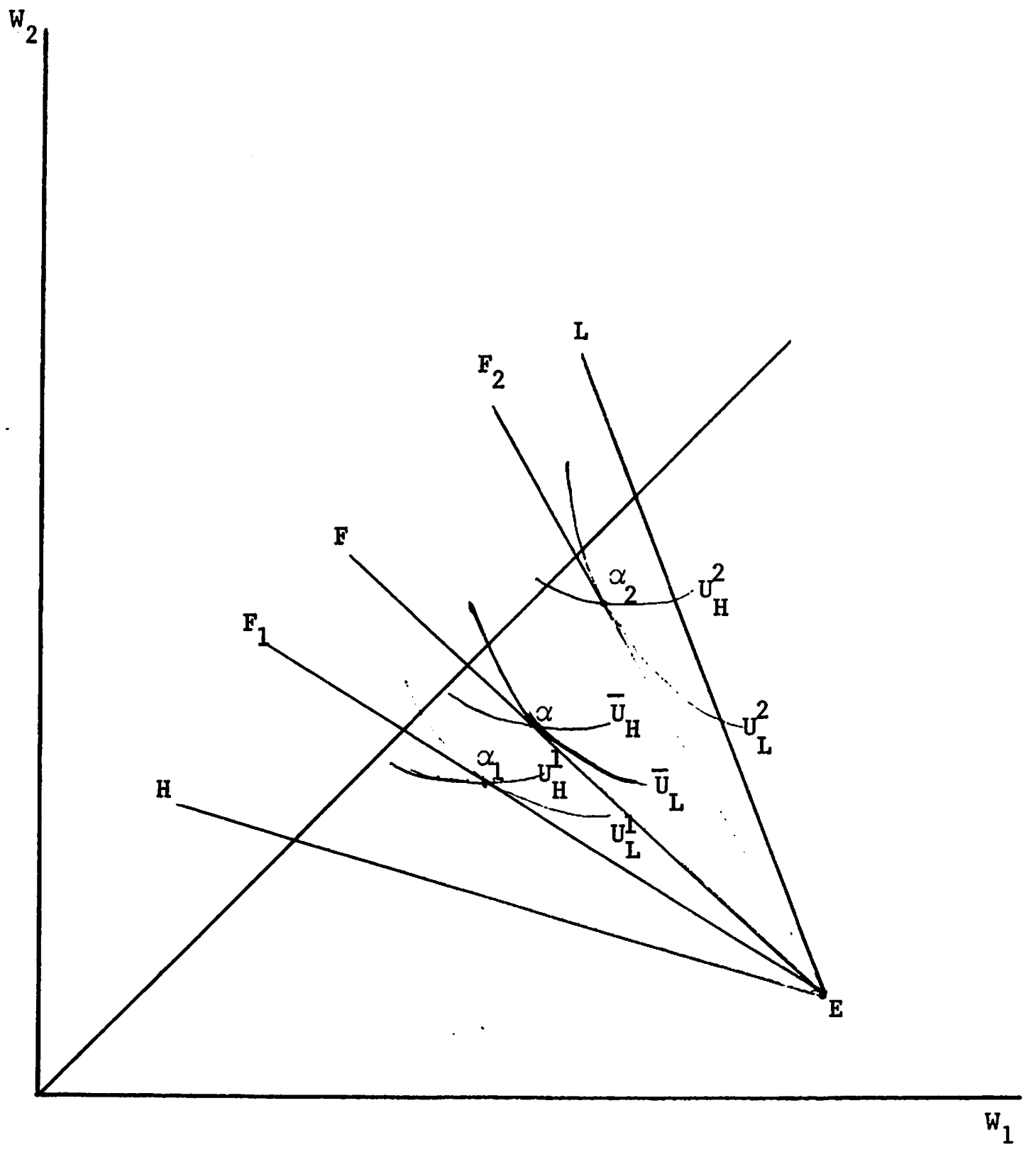


The following symbols are used to derive the results in section III.

index $i$

index $\mathbf{j}$

$$
w_{1}^{j}, w_{2}^{j}
$$

$\mathrm{w}_{1}, \mathrm{w}_{2}$

$\pi_{i}$

$\mathrm{P}_{\mathbf{i}}$

d

$$
\mu_{i}
$$

$\mathbf{A}, \mathrm{A}_{\mathbf{j}}$

$\mathbf{r}_{,} \mathbf{r}_{\mathbf{j}}$

$\mathbf{P}, \mathbf{P}_{\mathbf{j}}$ refers to risk type: $i=1$ for high risk type; $i=2$ for low risk type

refers to risk category: $j=1$ for high risk category; $j=2$ for low risk category

wealth in states 1 and 2 respectively for members of risk category $j$

wealth in states 1 and 2 respectively for the aggregate pool (before categorization)

refers to population of risk type $i$ probability of loss for risk type $1\left(p_{1}>p_{2}\right)$

loss (if state 2 is incurred)

expected loss for risk type $1\left(\mu_{1}=p_{1} d\right)$

actuarially fair pooled odds price for full coverage for the aggregate pool and for risk category $\mathrm{j}$ respectively

coverage level for aggregate pool and for risk category $j$ respectively (subscript $j$ is excluded when no confusion is likely)

actuarially fair price (for coverage level $\mathbf{r}$, $r_{j}$ ) for aggregate pool and risk category $j$ respectively

So, $P=r A$ and $P_{j}=r_{j} A_{j}$.

$\mathrm{U}(\mathrm{W})$

elementary (risk averse) utility function;

individuals are identical

The following relates to the categorization scheme and its relationship to the population of low and high risk types.

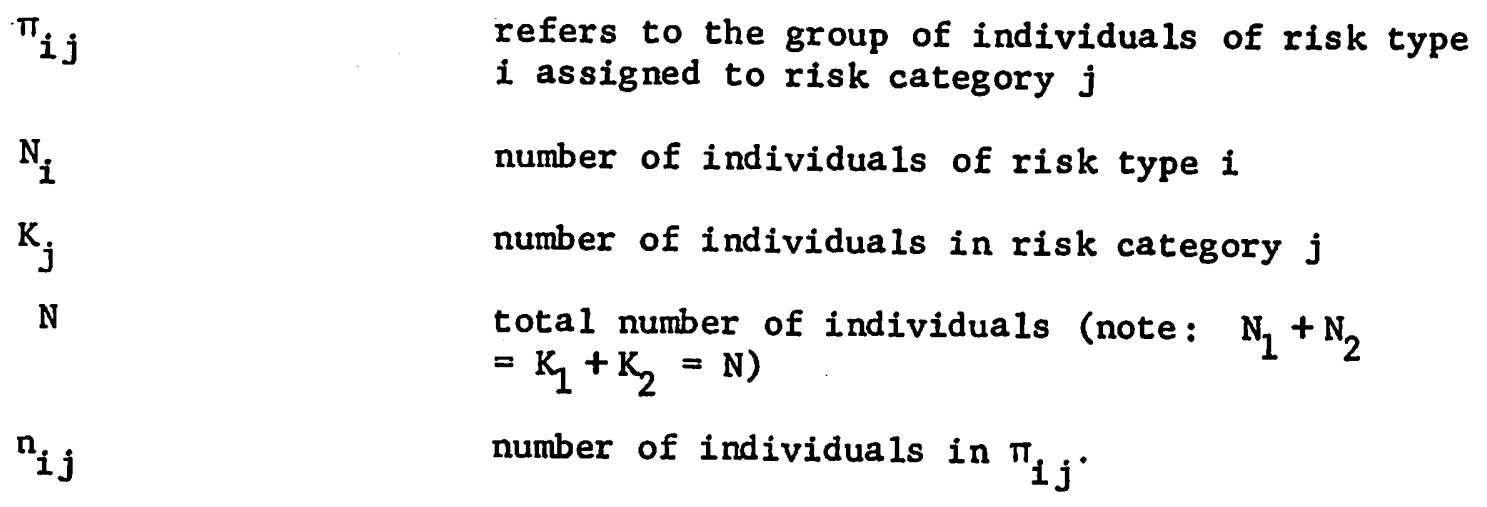


So,

$$
\begin{array}{ll}
\mathrm{n}_{11}+\mathrm{n}_{12}=\mathrm{N}_{1} & \mathrm{n}_{11}+\mathrm{n}_{21}=\mathrm{k}_{1} \\
\mathrm{n}_{21}+\mathrm{n}_{22}=\mathrm{N}_{2} . & \mathrm{n}_{22}+\mathrm{n}_{12}=\mathrm{k}_{2}
\end{array}
$$

$$
\begin{array}{r}
\bar{q}_{i}\left(=\frac{N_{i}}{N}\right) \\
q_{i j}\left(=\frac{n_{i j}}{K_{j}}\right)
\end{array}
$$

proportion of individuals of risk type $i$ in aggregate population

proportion of individuals of risk type $i$ in category $j$ (e.g., $q_{12}$ is the proportion of high risk types in the low risk category).

So,

$$
\begin{aligned}
& \bar{q}_{1}+\bar{q}_{2}=1 \\
& q_{22}+q_{12}=1 \\
& q_{11}+q_{21}=1
\end{aligned}
$$

e

is a parameter which characterizes the "information value" or "extent" of a categorization scheme $(\epsilon>0)$

where

$$
q_{11}=\bar{q}_{1}+\epsilon
$$

According to equation (2.3), the proportion of high risk types is greater in the high risk category than in the aggregate pool. It can be shown that $q_{11}=\bar{q}_{1}+\epsilon, \varepsilon \geq 0$ implies $q_{22}>\bar{q}_{2}$ (i.e., the categorization scheme also leads to a higher proportion of low risk types in the low risk category than in the aggregate pool). This result is demonstrated by Proposition 1 below (for proof, see Appendix).

Proposition 1. If $\mathrm{q}_{11}=\overline{\mathrm{q}}_{1}+e, \epsilon>0$, then
(i) $q_{22}=\bar{q}_{2}+\epsilon k$
(ii) $\mathrm{q}_{12}=\overline{\mathrm{q}}_{1}-\epsilon \mathrm{k}$
(iii) $q_{21}=\bar{q}_{2}-\epsilon, \quad$ where $k=\frac{k_{1}}{k_{2}}$. 
The full coverage $(r=1)$ actuarial fair price for members in the aggregate pool is $A=\bar{q}_{1} \mu_{1}+\bar{q}_{2} \mu_{2}$. This price is equal to the expected loss for an individual selected at random from the aggregate pool. It is the expected loss of a high risk individual $\left(\mu_{1}\right)$ maltiplied by the probability that an individual belonging to the aggregate pool is a high risk type $\left(\bar{q}_{1}\right)$ plus the expected loss of a low risk type $\left(\mu_{2}\right)$ multiplied by the probability that an individual belonging to the aggregate pool is a low risk type $\left(\bar{q}_{2}\right)$. Similarly, $A_{1}=q_{11} \mu_{1}+q_{21} \mu_{2}$ and $A_{2}=q_{12} \mu_{1}+q_{22} \mu_{2}$ are the actuarial costs of $100 \%$ coverage $\left(r_{1}, r_{2}=1\right)$ for the high and low risk categories respectively. Therefore :

$$
\begin{aligned}
& P=r\left(\bar{q}_{1} \mu_{1}+\bar{q}_{2} \mu_{2}\right) \\
& P_{1}=r_{1}\left(q_{11} \mu_{1}+q_{21} \mu_{2}\right) \\
& P_{2}=r_{2}\left(q_{12} \mu_{1}+q_{22} \mu_{2}\right)
\end{aligned}
$$

Using the results of proposition 1 we can write

$$
\begin{aligned}
& P=r A, \quad A=\bar{q}_{1} \mu_{1}+\bar{q}_{2} \mu_{2} \\
& P_{1}=r_{1} A_{1}, \quad A_{1}=\left(\vec{q}_{1}+\epsilon\right) \mu_{1}+\left(\bar{q}_{2}-\epsilon\right) \mu_{2} \\
& P_{2}=r_{2} A_{2}, \quad A_{2}=\left(\bar{q}_{1}-\epsilon k\right) \mu_{1}+\left(\bar{q}_{2}+\epsilon k\right) \mu_{2}
\end{aligned}
$$

\section{CATEGORIZATION AND INCOME INEQUALITY}

In this section it is demonstrated that under the assumption of a constant coinsurance rate ${ }^{6}$ (i.e., $r=r_{1}=x_{2}$ ) the Lorenz curve for the distribution of wealth before categorization will lie above (i.e., possess less inequality than) that for the distribution of wealth after categorization. of course, one does not generally expect the coinsurance rate chosen by consumers to be independent of the price of insurance. This assumption simply provides a 
benchmark case as more general results are not easily characterized. However, the results in this section are in a weak sense robust. In particular, a slight perturbation of this assumption (i.e., that $r=x_{1}=r_{2}$ ) leaves the results of this section unchanged. Furthermore, under more usual demand conditions categorization does not naturally lead to an unambiguous improvement in the post-categorization distribution relative to the constant coinsurance case.

In order to derive results concerning the distribution of wealth before and after categorization these situations must be characterized. The situation before categorization is quite straightforward. ${ }^{7}$ There are $\mathrm{N}_{1}$ high risk types who incur loss $d$ with probability $p_{1}$. Therefore, $p_{1} N_{1}$ of the high risk types incur loss $d$ and receive wealth $W_{2}=W-r A+r d-d$. There are $\mathrm{N}_{2}$ low risk types who incur loss d with probability $\mathrm{p}_{2}$. Therefore, $\mathrm{P}_{2} \mathrm{~N}_{2}$ of the low risk types incur loss $\mathrm{d}$ and also receive wealth $\mathrm{W}_{2}$. Hence, the total number of individuals who receive $\mathrm{w}_{2}$ (before categorization) is $\mathrm{p}_{1} \mathrm{~N}_{1}+\mathrm{p}_{2} \mathrm{~N}_{2}$. In a similar fashion it follows that $\left(1-\mathrm{p}_{1}\right) \mathrm{N}_{1}+\left(1-\mathrm{p}_{2}\right) \mathrm{N}_{2}$ individuals do not incur loss $d$ and, therefore, receive wealth $W_{1}=W-r A$. Using the results from equations (2.1) we get the following characterization of the pre-categorisation distribution.

\section{Before Categorization}

$$
\begin{array}{ll}
p_{1}\left(n_{11}+n_{12}\right)+p_{2}\left(n_{21}+n_{22}\right) & =s_{1}^{B} \text { individuals receive } w_{2} \\
\left(1-p_{1}\right)\left(n_{11}+n_{12}\right)+\left(1-p_{2}\right)\left(n_{21}+n_{22}\right) & =s_{2}^{B} \text { individuals receive } w_{1}
\end{array}
$$

The characterization of the distribution of wealth after categorization is slightly more complicated. This is because there are high and low risk types belonging to both the high and low risk categories. For example, there are $n_{11}$ high risk types in the high risk category with probability $p_{1}$ of receiving loss $d$. Therefore, $p_{1} n_{11}$ high risk types in the high risk category incur loss $d$ and 
receive wealth $w_{2}^{1}=w-r A_{1}+r d-d$. Similarly, $p_{2} n_{21}$ low risk types

assigned to the high risk category also incur loss $d$ and receive wealth $w_{2}^{1}$. It also follows that there are $\left(1-\mathrm{p}_{1}\right) \mathrm{n}_{11}$ high risk types in the high risk category who do not incur $108 \mathrm{~d}$, as well as $\left(1-\mathrm{p}_{2}\right) \mathrm{n}_{21}$ low risk types. These individuals receive wealth $w_{1}^{1}=w-r A_{1}$.

The wealth received by members of the low risk category is $\mathrm{w}_{2}^{2}=\mathrm{W}-\mathrm{rA}_{2}+\mathrm{rd}-\mathrm{d}$ if loss $\mathrm{d}$ is incurred and $\mathrm{w}_{1}^{2}=\mathrm{W}-\mathrm{rA}$ if not. The numbers of individuals receiving these levels of wealth can be derived in a similar fashion as for members of the high risk category. The results are summarized below.

\section{After Categorization}

$$
\begin{array}{ll}
p_{1} n_{11}+p_{2} n_{21} & =s_{1}^{A} \text { individuals receive } w_{2}^{1} \\
p_{1} n_{12}+p_{2} n_{22} & =s_{2}^{A} \text { individuals receive } w_{2}^{2} \\
\left(1-p_{1}\right) n_{11}+\left(1-p_{2}\right) n_{21} & =s_{3}^{A} \text { individuals receive } w_{1}^{1} \\
\left(1-p_{1}\right) n_{12}+\left(1-p_{2}\right) n_{22} & =s_{4}^{A} \text { individuals receive } w_{1}^{2}
\end{array}
$$

The Lorenz curve description of a wealth distribution traces the percentage of total wealth attained by the "poorest" $\rho$ percent as $\rho$ increases from zero to one. One must therefore be able to order the individuals according to wealth $w_{i}, i=1, \ldots, N$ with $w_{1} \leq w_{2} \leq \ldots \leq w_{N}$. Since only partial insurance is purchased in any of the pooling equilibria $(0<r<1)$ it follows that before categorization ${ }^{8} W_{2}<W_{1}$ and after categorization $w_{2}^{1}<w_{1}^{1}$ and $w_{2}^{2}<w_{1}^{2}$. Also, since $A_{2}<A<A_{1}$, it follows that $w_{2}^{1}<w_{2}<w_{2}^{2}$ and $w_{1}^{1}<w_{1}<w_{1}^{2}$; that is, comparing the levels of wealth for individuals both before and after categorization we find that members of the low risk category who do not incur loss a receive the highest level of wealth while members of the high risk category who do incur 
loss $d$ receive the lowest level of wealth. One cannot, however, determined whether $w_{2}^{2} \gtreqless w_{1}^{1}$. In the following discussion of the implications of categorization on the Lorenz depiction of the distribution of wealth it is assumed that $W_{2}^{2} \leq w_{1}^{1}$. However, both cases are illustrated and considered in the proof provided in the appendix.

Consider the wealth distributions presented in equations (3.1) to (3.6). Before categorization, the poorest $s_{1}^{B}$ individuals incur loss $d$ and receive wealth $W_{2}$ while the next $s_{2}^{B}$ individuals do not incur loss $d$ and so receive wealth $W_{1}$. After categorization, the first $S_{1}^{A}$ individuals are the poorest, receiving wealth $W_{2}^{1}$ while, it is assumed here, the next worse off group (numbering $s_{2}^{A}$ ) receive $W_{2}^{2}$. The next $s_{3}^{A}$ individuals receive $W_{1}^{1}$ and the remaining $s_{4}^{A}$ individuals are best off receiving $w_{1}^{2}$.

Total wealth after categorization is equal to that before categorization. This result follows intuitively since in both cases firms earn zero expected profits and are faced with zero administrative costs. Therefore, insurance simply redistributes wealth from individuals in the good state (not incurring loss d) to those in the bad state (incurring loss d). No withdrawals or injections occur.

The Lorenz curve ${ }^{9}$ for the wealth distribution before categorization is depicted by line $B$ in Figure II. The first $s_{1}^{B}$ individuals receive wealth $\mathrm{W}_{2}$ and the next $\mathrm{s}_{2}^{\mathrm{B}}$ individuals receive wealth $\mathrm{W}_{1}$. Since $\mathrm{w}_{2}<\mathrm{w}_{1}$ the Lorenz curve rises less steeply over the first $s_{1}^{B}$ individuals than for the next $\mathrm{s}_{2}^{\mathrm{B}}$ individuals.

The Lorenz curve for the wealth distribution after categorization is represented by line $A$ in Figure II. Since the first $s_{1}^{A}$ individuals receive wealth $W_{2}^{1}$, which is less than $W_{2}$, the Lorenz curve over this interval lies 
below the one before categorization. The next $s_{2}^{A}$ individuals receive wealth $\mathrm{W}_{2}^{2}>\mathrm{W}_{2}$ so that the $A$ curve rises more quickly over this interval than does the B curve. However, the percentage of total wealth obtained by the first $s_{1}^{A}+s_{2}^{A}\left(=s_{1}^{B}\right)$ individuals never "catches up" to the percentage of total wealth obtained over the same interval before categorization. The intuitive explanation of this result is that the poorest $s_{1}^{B}$ individuals in each case are those who incur loss $\mathrm{d}$. High risk types are both more likely to be assigned to the high risk category and are also more likely to incur loss d. Therefore, since the impact of categorization is to increase the price of insurance to members of the high risk category, these individuals incur a proportionately greater reduction in wealth when incurring loss d than do the poorest $\mathrm{s}_{1}^{\mathrm{B}}$ individuals before categorization.

The next $s_{3}^{A}$ individuals after categorization do not incur loss $d$ but do face a higher price of insurance relative to the situation before categorization, since they have been assigned to the high risk category. Over this interval the post-categorization Lorenz curve (A) rises less steeply than does the pre-categorization one (B). The remaining $\mathrm{s}_{4}^{\mathrm{A}}$ individuals do not incur loss d and, after categorization, are faced with a lower price of insurance. Over this interval the "A" Lorenz curve rises more rapidly than does the "B" one and it finally "catches up".

As mentioned earlier, it is possible that $w_{1}^{1}>w_{2}^{2}$; that is, members of the high risk category who do not incur loss d may be better off than members of the low risk category who do incur loss d. As is seen below, if this is the case then the Lorenz curve characterization of the wealth distribution after categorization becomes slightly more complicated. Since the two poorest groups of individuals after categorization receive less than they would if they 
Figure II

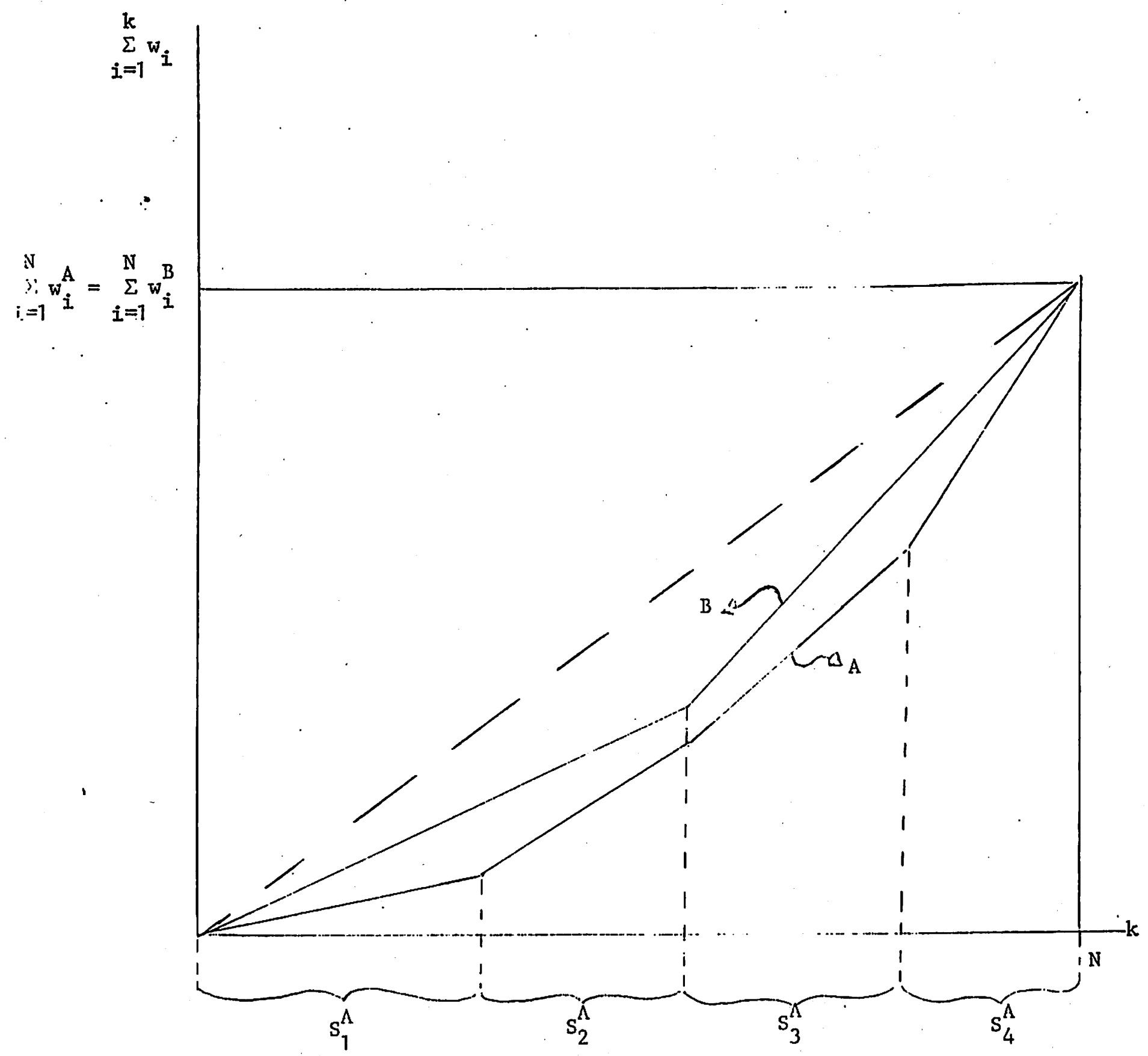


received $w_{2}^{1}$ and $w_{2}^{2}$ respectively, the Lorenz curve for these groups lies below the one before categorization. The slight complication is that it is no longer necessarily the case that the number of individuals in the poorest two groups after categorization is equal to the number of individuals in the poorest group before categorization. That is, it may be that $s_{1}^{A}+s_{2}^{A}>s_{1}^{B}$ or $s_{1}^{A}+s_{2}^{A}<s_{1}^{B}$. Nevertheless, it can be shown (see the proof in the appendix) that the Lorenz curve after categorization will lie below the Lorenz curve before categorization for these cases also. This is demonstrated in Figures III and IV.

Theorem 1. Suppose that the quantity of insurance (i.e., the coinsurance rate) purchased before and after categorization is the same (and equal to $r$ ). Iet $w_{i}^{B}, i=1, \ldots, N$ and $w_{i}^{A}, i=1, \ldots, N$ represent the wealth distribution for the precategorization and post-categorization situations respectively. Order these vectors so that $w_{1}^{B} \leq \cdots \leq w_{N}^{B}$ and $w_{1}^{A} \leq \cdots \leq w_{N}^{A}$. Then $\sum_{i=1}^{k} w_{i}^{B} \geq \sum_{i=1}^{k} w_{i}^{A}$ for all $k \leq N$ (with at least one strict inequality) and $\sum_{i=1}^{N} w_{i}^{B}=\sum_{i=1}^{N} w_{i}^{A}$. That is, by the Lorenz criterion the wealth distribution before categorization is "more equal" than the wealth distribution after categorization.

As is seen in Figures II, III, and IV, the Lorenz curve for the postcategorization case lies strictly below that for the pre-categorization one. This suggests that at least "sma11" perturbations away from the assumption that the coinsurance rate is uninfluenced by changes in the price of insurance can be allowed without affecting the results of theorem 1 . Furthermore, suppose demand is such that the coinsurance rate varies inversely with the price. Then $r_{1}$, coverage for members of the high risk category will be less than $r$ while $x_{2}$, coverage for members of the 1 ow risk category will be greater 
Figure III

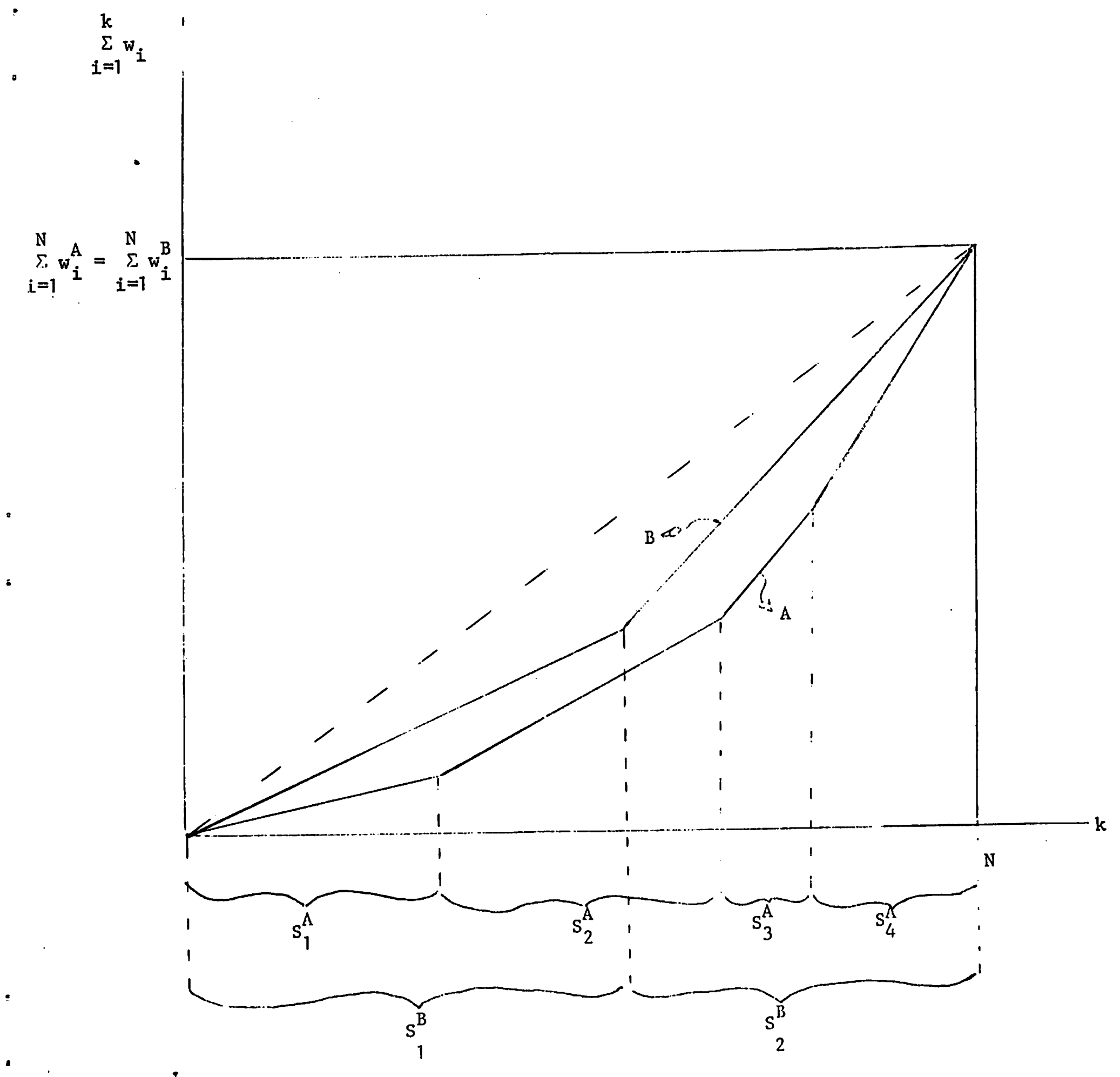


Figure IV

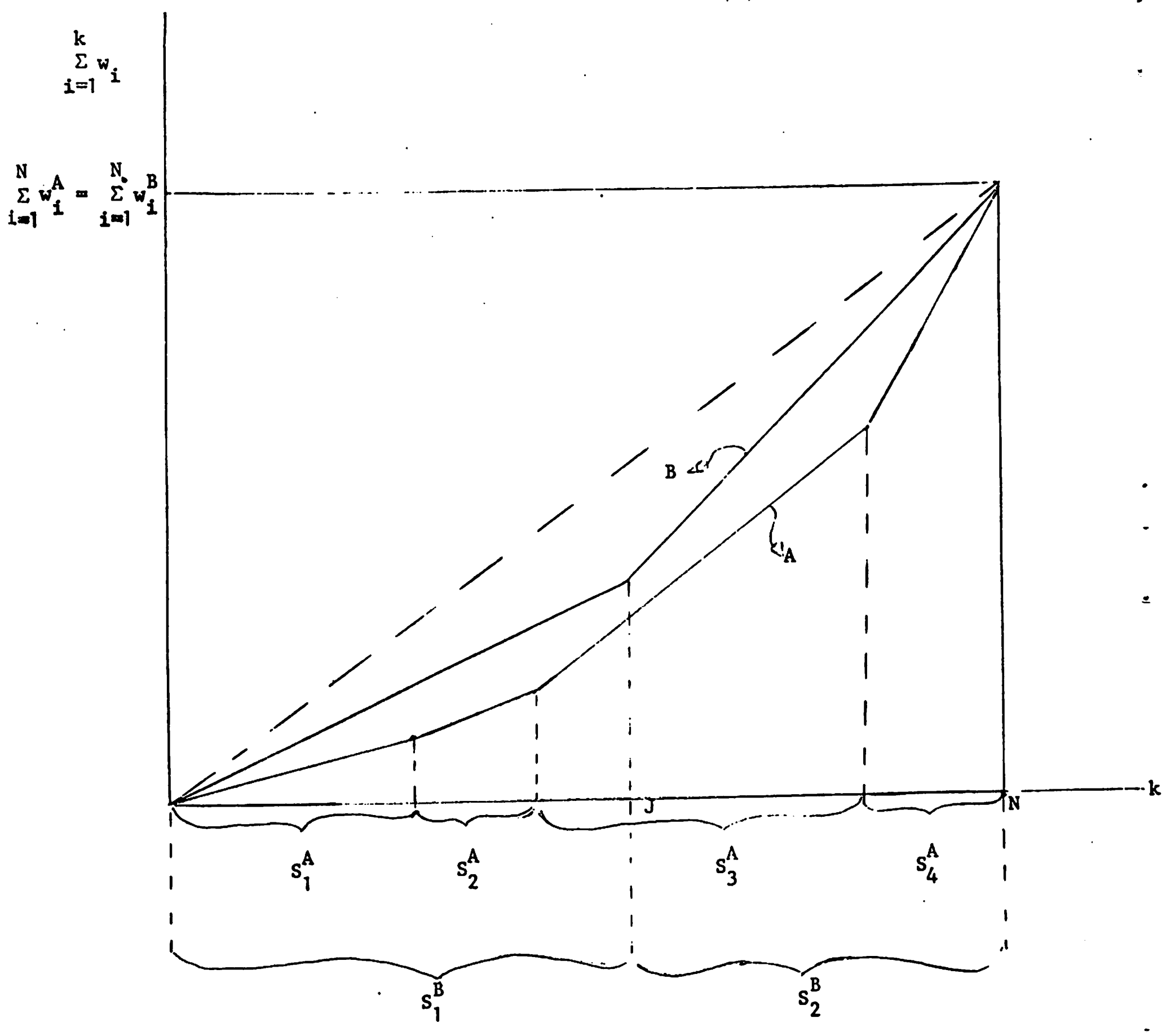


than $r$. The result is that $w_{2}^{1}$ (wealth of individuals in the high risk category who incur loss d) will be smaller than for the case where the coinsurance rate is held constant. This implies that the first section (i.e., over the interval $s_{1}^{A}$ ) of the post-categorization Lorenz curve will lie below that for the "A" curve in any of Figures II, III, and IV and hence, a fortiori, will lie below the pre-categorization curve. Although the remainder of the post-categorization Lorenz curve need not lie everywhere below that for the pre-categorization one, it does not follow that the adoption of the more usual demand assumption will naturally lead to a preferred distribution of wealth resulting from categorization (i.e., relative to the constant coinsurance case). Therefore, the result of theorem 1 is robust in a weak sense.

\section{CONSUMER PREFERENCES FOR}

CATEGORIZATION SCHEMES

It is sometimes argued that the effect of categorization should not be analyzed using the "value laden" approach of comparing income distributions. Instead it can be argued that without categorization the presence of high risk types imposes an externality on low risk types since, unlike the perfect information case, the low risk types cannot be offered full insurance at their actuarially fair rates. ${ }^{10}$ Therefore, any policy which eliminates or reduces this externality is a "fair" one and ought to be implemented regardless of any adverse distributional effects. Imperfect categorization is such a policy (or process) in that it allows a greater percentage of low risk types to be offered a price which more closely reflects the costs which they impose on firms. 
However, if one were to ask individuals before they are assigned to their respective risk categories if they would prefer that some particular categorization scheme not be implemented, the response may be unanimously positive. That is, even low risk types, who have a larger probability than do high risk types of obtaining the advantageous outcome of being assigned to the low risk category, may. prefer the status quo (i.e., no categorization). Although the opposite may also be true, this result nevertheless demonstrates that the use of information which reduces the adverse effects of price discrimination may, ex ante, make even the "apparently advantaged" group worse off.

In order to investigate the issues introduced in this section, more notation as well as some assumptions concerning expectations must be presented. Let $\mathrm{VW}_{i}$ represent the expected utility that a risk type $i$ individual receives from the anticipation of some particular prospective categorization scheme which he believes will be implemented. Let $v_{i}$ represent his utility if the status quo (i.e., no categorization) is preserved. If $v_{i}<v_{i}$ then the individual prefers that the categorization scheme not be implemented, while if $\mathrm{V}_{i}>\mathrm{v}_{i}$ then he does prefer that it be implemented.

Let $\mathrm{V}_{i j}$ represent the expected utility of a risk type $i$ individual who is assigned to risk category $j$ and let $p_{i j}$ be the probability that an individual of risk type $i$ is assigned to category $j$. Then

$$
\begin{aligned}
& w_{1}=p_{11} v_{11}+p_{12} v_{12} \\
& v_{2}=p_{21} v_{21}+p_{22} v_{22}
\end{aligned}
$$

The expected utility of a high risk type, given that he does not yet know the risk category to which he will be assigned, is equal to the probability that he will be assigned to the high risk category $\left(\mathrm{p}_{11}\right)$ multiplied by the expected utility he would receive if he were $\left(v_{11}\right)$, plus the probability that he will be 
assigned to the low risk category $\left(\mathrm{p}_{12}\right)$ multiplied by the expected utility he would receive if he were $\left(\mathrm{V}_{12}\right)$. A similar explanation can be offered for equation (4.2).

We must now introduce an assumption concerning expectations. Since individuals know their own risk type it would be inappropriate to assume that all individuals hold the same probabilities of being assigned to the two risk categories. Instead, it is assumed that the probabilities, held by individuals, of being assigned to the two risk categories are substantiated by the actual frequency experience for the members of each risk type. Therefore, $p_{i j}=\frac{n_{i j}}{N_{i}}$; that is, the probability that an individual of risk type $i$ holds of being assigned to category $j$ is equal to the actual number of risk type $i$ individuals that would be assigned to category $j$ (if the prospective scheme were implemented) divided by the total number of individuals of risk type $i$.

Since $p_{i j}=\frac{n_{i j}}{N_{i}}$ and $q_{i j}=\frac{n_{i j}}{k_{j}}$ it follows that $p_{i j}=q_{i j} \frac{k_{j}}{N_{i}}$. Using the results of Proposition 1 we get the following equalities.

$$
\begin{aligned}
& \mathrm{p}_{11}=\left(\overline{\mathrm{q}}_{1}+\epsilon\right) \frac{\mathrm{K}_{1}}{\mathrm{~N}_{1}} \\
& \mathrm{p}_{12}=\left(\overline{\mathrm{q}}_{1}-\epsilon \mathrm{k}\right) \frac{\mathrm{K}_{2}}{\mathrm{~N}_{1}} \\
& \mathrm{p}_{21}=\left(\overline{\mathrm{q}}_{2}-\epsilon\right) \frac{\mathrm{K}_{1}}{\mathrm{~N}_{2}} \\
& \mathrm{p}_{22}=\left(\overline{\mathrm{q}}_{2}+\epsilon \mathrm{k}\right) \frac{\mathrm{K}_{2}}{\mathrm{~N}_{2}}
\end{aligned}
$$

It is shown in the appendix (see Proposition 2) that $\mathrm{p}_{22}>\mathrm{p}_{12}$; that is, the probability of being assigned to the low risk category is greater for low risk types than for high risk types. Similarly, $p_{11}>p_{21}$. Furthermore, 
it is easy to show that the expected price of full coverage insurance offered to low risk types as a result of categorization is less than the price with no categorization (i.e., $\mathrm{P}_{21} \mathrm{~A}_{1}+\mathrm{P}_{22} \mathrm{~A}_{2}>\mathrm{A}$ ). In this sense, the imperfect categorization of risks leads to a reduction in the average level of price discrimination. However, as previously stated, even though the reduction in price discrimination is "apparently" to the advantage of low risk types, they may nevertheless desire that a categorization scheme not be implemented.

The possible outcome that all individuals may prefer that a prospective categorization scheme not be implemented can be demonstrated by the following example. The parameters and results for this example are listed in Table I below. Before categorization, high risk types ex ante utility is $\mathrm{v}_{1}=-0.2477 \times 10^{-9}$ while low risk types utility is $\mathrm{v}_{2}=-0.1094 \times 10^{-9}$. The particular categorization scheme chosen has $\mathrm{K}_{1}=20$ (the number of individuals in the high risk category), $\mathrm{K}_{2}=80$ (the number of individuals assigned to the low risk category) and $\epsilon=0.3$. It follows from equations (4.3) to (4.6), as well as the parameters $\bar{q}_{1}=0.3, \bar{q}_{2}=0.7, N_{1}=30$ and $N_{2}=70$, that $p_{11}=0.4$, $\mathrm{P}_{12}=0.6, \mathrm{p}_{22}=0.886$, and $\mathrm{p}_{21}=0.114$. Using the results for $\mathrm{V}_{i j}$ from Table $\mathrm{I}$ we get

$$
\begin{aligned}
& \mathrm{vv}_{1}=0.4 \mathrm{v}_{11}+0.6 \mathrm{v}_{12}=-0.7660 \times 10^{-9} \\
& \mathrm{v}_{2}=0.114 \mathrm{~V}_{21}+0.886 \mathrm{~V}_{22}=-0.1255 \times 10^{-9}
\end{aligned}
$$

Since $\mathrm{VV}_{1}<\mathrm{v}_{1}$ and $\mathrm{VV}_{2}<\mathrm{v}_{2}$ it follows that both high and low risk types receive a lower level of utility when contemplating this particular categorization scheme than they do under the status quo of no categorization. Therefore, if individuals could choose (ex ante to knowing the category to which they will be assigned) whether or not to have a particular categorization. scheme implemented, they may unanimously reject it. If this were the case one 
TABLE I

Parameters

$$
\begin{aligned}
& \text { utility function } U(W)=\frac{W^{\gamma}}{\gamma}, \quad \gamma=-5 \\
& W=100, d=99, P_{1}=0.8, P_{2}=0.1
\end{aligned}
$$

(before categorization)

$$
\mathrm{N}_{1}=30, \mathrm{~N}_{2}=70, \bar{q}_{1}=0.3, \bar{q}_{2}=0.7
$$

(after categorization)

$$
\begin{aligned}
& \mathrm{K}_{1}=20, \mathrm{~K}_{2}=80, \mathrm{k}=0.25, \epsilon=0.3 \\
& \mathrm{q}_{11}=0.6, \mathrm{q}_{21}=0.4, \mathrm{q}_{22}=0.775, \mathrm{q}_{12}=0.225
\end{aligned}
$$

\section{Results}

(before categorization)

$$
\begin{aligned}
& A=30.69, r=0.845, W_{1}=74.1, W_{2}=58.7 \\
& U\left(W_{1}\right)=-0.8964 \times 10^{-10} ; U\left(W_{2}\right)=-0.2872 \times 10^{-9} \\
& \mathrm{~V}_{1}=\left(1-\mathrm{p}_{1}\right) \mathrm{U}\left(\mathrm{W}_{1}\right)+\mathrm{p}_{1} \mathrm{U}\left(\mathrm{W}_{2}\right)=-0.2477 \times 10^{-9} \\
& \mathrm{~V}_{2}=\left(1-\mathrm{p}_{2}\right) \mathrm{U}\left(\mathrm{W}_{1}\right)+\mathrm{p}_{2} \mathrm{U}\left(\mathrm{W}_{2}\right)=-0.1094 \times 10^{-9}
\end{aligned}
$$

high risk category:

$$
\begin{aligned}
& A_{1}=51.48, r_{1}=0.815, W_{1}^{1}=58.04, W_{2}^{1}=39.72 \\
& U\left(W_{1}^{1}\right)=-0.3033 \times 10^{-9}, U\left(W_{2}^{1}\right)=-0.2023 \times 10^{-8} \\
& V_{11}=\left(1-p_{1}\right) U\left(W_{1}^{1}\right)+P_{1} U\left(w_{2}^{1}\right)=-0.1679 \times 10^{-8} \\
& V_{21}=\left(1-p_{2}\right) U\left(W_{1}^{1}\right)+p_{2} U\left(w_{2}^{1}\right)=-0.4753 \times 10^{-9}
\end{aligned}
$$

low risk category:

$$
\begin{aligned}
& A_{2}=25.49, r_{2}=0.864, w_{1}^{2}=77.97, w_{2}^{2}=64.51 \\
& U\left(W_{1}^{2}\right)=-0.6937 \times 10^{-10}, U\left(w_{2}^{2}\right)=-0.1791 \times 10^{-9} \\
& V_{12}=\left(1-p_{1}\right) U\left(w_{1}^{2}\right)+p_{1} U\left(w_{2}^{2}\right)=-0.1572 \times 10^{-9} \\
& V_{22}=\left(1-p_{2}\right) U\left(w_{1}^{2}\right)+p_{2} U\left(w_{2}^{2}\right)=-0.8035 \times 10^{-10} \\
& \text { Note: } v_{11}<v_{1}, v_{21}<v_{2}, v_{12}>v_{1}, v_{22}>v_{2} .
\end{aligned}
$$


might expect consumers to opt for longer-term contracts with fixed premiums. However, if for some reason long-term contracts are infeasible, political pressure to suppress the use of imperfect information for categorizing. risks may arise.

\section{CONCLUSIONS}

If, after categorization, the quantity of insurance (as measured by the coinsurance rate) offered to members of both risk categories is the same as that before categorization then, according to the Lorenz criterion, the distribution of wealth after categorization is more unequal than that for the situation before categorization. ${ }^{11}$ If one assumes full interpersonal comparability of utility, with $U_{i}(\cdot)=U(\cdot)$ for all $i$, then the results of Dasgupta, et al. [1973] can be employed here to show that a reduction in social welfare is associated with the imperfect categorization of risks. This result holds for the assumptions made in this paper (including the constant coinsurance assumption) for any symmetric and quasiconcave social welfare function.

However, the issue of categorizing risks when different risk types are present in the population can instead be considered from the point of view of price discrimination. The use of imperfect information to categorize risks leads to a reduction in the average level of price discrimination faced by consumers. In particular, the expected price of insurance for low risk types with categorization is less than that without categorization. Therefore, the result of categorization is that the average price of insurance will more closely reflect the particular costs imposed on firms by each risk type in such a way that "vertical" price discrimination is reduced. In 
terms of the issue of price discrimination one might then argue that, despite any adverse distributional effects, the use of imperfect categorization is "fair" and ought to be encouraged. However, as is shown in this paper this conclusion appears somewhat paradoxical. The reason for this claim is that even those individuals who from the point of view of price discrimination,are favoured by categorization may prefer that the future use of such information be suppressed. 


\section{Footnotes}

${ }^{1}$ See Section II for a definition of the Wilson $E 2$ equilibrium.

2 A pooling contract is one in which both high and low risk types purchase the same policy at a price which reflects the actuarially fair pooled cost of providing insurance. This price is the weighted average of the expected value of losses for high and low risk types, the weights being the proportion of high and low risk types respectively.

${ }^{3}$ In this context price discrimination occurs when different groups of consumers are not charged prices which reflect their expected costs (i.e., when there is some cross-subsidization). Imperfect categorization can then be viewed as an attempt to reduce vertical inequity. For a discussion of this issue see Etgar [1975].

${ }^{4}$ Under the usual Nash assumption firms do not possess this foresight. For a more lengthy discussion of the Wilson E2 equilibrium see Hoy [1981] or Wilson [1976].

${ }^{5}$ It is assumed in this paper that all equilibria are of the pooling type. The requirement for a Wilson $\mathrm{E} 2$ equilibrium to be a pooling one is that the proportion of high risk types be less than some critical level.

${ }^{6}$ Since the effect of categorization is to change the price of insurance for members of the two risk categories this assumption implies that the demand for insurance, with quantity defined as the coinsurance rate, is price inelastic. In a paper by Hoy and Robson [1981] it is shown that if consumers display constant relative risk aversion then it is possible, although unlikely, that insurance may be Giffen. It is, therefore, also possible that $r$ will not change as price changes. 
${ }^{7}$ It is assumed that all probabilities are realized in terms of actual ex post frequencies.

${ }^{8}$ For example, the wealth distribution before categorization has $w_{i}=w_{2}, i=1, \ldots, s_{1}^{B}$ and $w_{i}=w_{1}, i=s_{1}^{B}+1, \ldots, N$.

${ }^{9}$ These Lorenz curves are not normalized in terms of percentages.

The discussion of the results is made clearer by not doing so.

${ }^{10}$ At least not by firms who must earn non-negative profits.

${ }^{11}$ Although this result is only in a weak sense robust with respect to the assumption of a constant coinsurance rate, it does provide a benchmark case from which to consider more general cases. 


\section{References}

Dasgupta, P., A. Sen and D. Starrett, "Notes on the Measurement of Inequality," Journal of Economic Theory, VI (1973), 180-7.

Etgar, M., "Unfair Price Discrimination in P-L Insurance and the Reliance on Loss Ratios," Journal of Risk and Insurance, XLII (Dec. 1975), 615-24.

Hoy, M., "Categorizing Risks in the Insurance Industry," Quarterly Journal of Economics, for thcoming (1981).

Hoy, M. and A. Robson, "Insurance as a Giffen Good," unpublished mimeo, University of Western Ontario, April 1981.

Wilson, C., "A Model of Insurance Markets with Asymmetric Information," Cowles Foundation Discussion Papers, No. 432, June 1976.

Department of Economics University of Western Ontario London, Canada 
Appendix

Proofs of the propositions and theorems are presented

be low.

Proposition 1

If $q_{11}=\bar{q}_{1}+\epsilon, \epsilon>0$, then

(i) $q_{22}=\vec{q}_{2}+\epsilon k$

(ii) $q_{12}=\bar{q}_{1}-\varepsilon k$

(iii) $q_{21}=\vec{q}_{2}-\epsilon$, where $k=\frac{k_{1}}{k_{2}}$

\section{Proof:}

$$
\begin{aligned}
& \mathrm{q}_{11}=\overline{\mathrm{q}}_{1}+\epsilon \\
\Rightarrow & \frac{\mathrm{n}_{11}}{\mathrm{~K}_{1}}=\frac{\mathrm{N}_{1}}{\mathrm{~N}}+\epsilon \\
\Rightarrow & \mathrm{n}_{11}\left(\mathrm{~N}_{1}+\mathrm{N}_{2}\right)=\left(\mathrm{N}_{1}+\mathrm{N} \epsilon\right)\left(\mathrm{n}_{11}+\mathrm{n}_{21}\right)
\end{aligned}
$$

(i.e., from equations $(2.1)$ and the fact that $N_{1}+N_{2}=N$ ). Upon rearranging the above and again using (2.1) it follows that

$$
\begin{aligned}
& \quad \frac{N_{1}-n_{12}}{n_{21}}=\frac{n_{21} N_{1}+N \in K_{1}}{n_{21} N_{2}} \\
& \Rightarrow \frac{N_{2}-n_{21}}{n_{12}}=\frac{N_{2}}{N_{1}}+\frac{N \in K_{1}}{N_{1} n_{12}} \text {, which with equations (2.1) } \\
& \Rightarrow n_{22}=\frac{n_{12} N_{2}+N \in K_{1}}{N_{1}} \\
& \text { or } n_{22} N_{1}=N_{2} n_{12}+N \in K_{1} \\
& \quad \text { Adding } n_{22} N_{2} \text { to both sides and using (2.1) again it follows that } \\
& \quad n_{22}=N_{2} K_{2}+N \in K_{1} \\
& \frac{n_{22}}{K_{2}}=\frac{N_{2}}{N}+\epsilon \frac{K_{1}}{K_{2}}
\end{aligned}
$$


i.e., $q_{22}=\bar{q}_{2}+$ ek and (i) is proved. Using the relationships in (2.2) it follows immediately that

$$
\begin{aligned}
& \text { (ii) } q_{12}=\bar{q}_{1}-\varepsilon k \\
& \text { (iii) } q_{21}=\bar{q}_{2}-\epsilon
\end{aligned}
$$

\section{Proposition 2}

Given that $p_{i j}=\frac{n_{i j}}{N_{i}}$, it follows that

(i) $\mathrm{p}_{22}>\mathrm{p}_{12}$ and

(ii) $\mathrm{p}_{11}>\mathrm{p}_{21}$.

\section{Proof:}

(i) from (4.6) and (4.4) it follows that

$$
\begin{aligned}
& \mathrm{p}_{22}=\left(\overline{\mathrm{q}}_{2}+\epsilon \mathrm{k}\right) \frac{\mathrm{K}_{2}}{\mathrm{~N}_{2}} \\
& \mathrm{p}_{12}=\left(\overline{\mathrm{q}}_{1}-\epsilon \mathrm{k}\right) \frac{\mathrm{K}_{2}}{\mathrm{~N}_{1}}
\end{aligned}
$$

$$
\text { So, } \begin{aligned}
p_{22}>p_{12} \\
\Leftrightarrow\left(\bar{q}_{2}+\epsilon k\right) \frac{k_{2}}{\bar{N}_{2}}>\left(\bar{q}_{1}-\epsilon k\right) \frac{k_{2}}{N_{1}}
\end{aligned}
$$$$
\Leftrightarrow \frac{\bar{q}_{2}+\epsilon k}{\bar{q}_{2}}>\frac{\bar{q}_{1}-\epsilon k}{\bar{q}_{1}} \text { (upon multiplication by } N \text { ) }
$$

$\Leftrightarrow 1+\frac{\varepsilon k}{\bar{q}_{2}}>1-\frac{\epsilon k}{\bar{q}_{1}}$ which holds since all parameters are positive.

(ii) can be shown in a similar fashion.

Theorem 1. Suppose that the quantity of insurance purchased before and after categorization is the same (and equal to $r$ ). Let $w_{i}^{B}, i=1, \ldots, N$ and $w_{i}^{A}, i=1, \ldots, N$ represent the wealth distribution for the pre-categorization and post-categorization situations respectively. $w_{1}^{A} \leq \ldots \leq w_{N}^{B}$. Then $\underset{i=1}{k} w_{i}^{B} \geq \sum_{i=1}^{k} w_{i}^{A}$ for all $k \leq N$ (with at least one strict 
inequality) and $\prod_{i=1}^{N} w_{i}^{B}=\dddot{i}_{i=1}^{N} w_{i}^{\Lambda}$. That is, by the Lorenz criterion the wealth distribution before categorization is "more equal" than the wealth distribution after categorization.

Proof: This theorem is proved in three parts. Part I shows that $\sum_{i=1}^{N} w_{i}^{B}=\sum_{i=1}^{N} w_{i}^{A}$, part II shows that $\sum_{i=1}^{k} w_{i}^{B} \geq \sum_{i=1}^{k} w_{i}^{A}$ for all $k$ when $w_{2}^{1}<w_{2}^{2} \leq w_{1}^{1}<w_{1}^{2}$, while part III shows the same result when $w_{2}^{1}<w_{1}^{1}<w_{2}^{2}<w_{1}^{2}$ (these are the only possible cases).

\section{$\underline{\text { Part I }}$}

$$
\begin{aligned}
& \text { Show that } \sum_{i=1}^{N} w_{i}^{B}=\sum_{i=1}^{N} w_{i}^{A} \text {. } \\
& \sum_{i=1}^{N} w_{i}^{B}=\left[p_{1}\left(n_{11}+n_{12}\right)+p_{2}\left(n_{21}+n_{22}\right)\right] w_{2} \\
& +\left[\left(1-p_{1}\right)\left(n_{11}+n_{12}\right)+\left(1-p_{2}\right)\left(n_{21}+n_{22}\right)\right] w_{1}
\end{aligned}
$$

Upon substitution of $W_{2}=W-r A+r d-d$ and $W_{1}=W-r A$ with $A=\bar{q}_{1} \mu_{1}+\bar{q}_{2} \mu_{2}$ we get

$$
\begin{aligned}
& \sum_{i=1}^{N} w_{i}^{B}=\left(n_{11}+n_{12}+n_{21}+n_{22}\right) w-\left(n_{11}+n_{12}+n_{21}+n_{22}\right) r\left(\bar{q}_{1} \mu_{1}+\bar{q}_{2} \mu_{2}\right) \\
& \quad-\left[p_{1}\left(n_{11}+n_{12}\right)+p_{2}\left(n_{21}+n_{22}\right)\right](1-r) d \\
& \sum_{i=1}^{N} w_{i}^{A}=\left[p_{1} n_{11}+p_{2} n_{21}\right] w_{2}^{1}+\left[p_{1} n_{12}+p_{2} n_{22}\right] w_{2}^{2} \\
& \quad+\left[\left(1-p_{1}\right) n_{11}+\left(1-p_{2}\right) n_{21}\right] w_{1}^{1}+\left[\left(1-p_{1}\right) n_{12}+\left(1-p_{2}\right) n_{22}\right] w_{1}^{2}
\end{aligned}
$$

Upon substitution of $w_{2}^{1}=w-r A_{1}+r d-d, w_{1}^{1}=w-r A_{1}, w_{2}^{2}=w-r A_{2}+r d-d$, and $w_{2}^{1}=w-r A_{2}$ with $A_{1}=\left(\bar{q}_{1}+\epsilon\right)_{\mu_{1}}+\left(\bar{q}_{2}-\epsilon\right)_{\mu_{2}}$ and $A_{2}=\left(\bar{q}_{1}-\epsilon k\right) \mu_{1}+\left(\bar{q}_{2}+\epsilon k\right)_{\mu_{2}}$ we

get

$$
\begin{aligned}
& \sum_{i=1}^{N} w_{i}^{A}=\left(n_{11}+n_{12}+n_{21}+n_{22}\right) w-\left(n_{11}+n_{12}+n_{21}+n_{22}\right) r\left(\bar{q}_{1} \mu_{1}+\bar{q}_{2} \mu_{2}\right) \\
& \quad-\left[p_{1}\left(n_{11}+n_{12}\right)+p_{2}\left(n_{21}+n_{22}\right)\right](1-r) d-r \in\left(n_{11}+n_{21}\right)\left(\mu_{1}-\mu_{2}\right) \\
& \quad+r \in k\left(n_{12}+n_{22}\right)\left(\mu_{1}-\mu_{2}\right)
\end{aligned}
$$


Recal1, $k=\frac{k_{1}}{k_{2}}, k_{1}=n_{11}+n_{21}$, and $k_{2}=n_{12}+n_{22}$. Iherefore, the last two terms are

$$
\begin{aligned}
& -r \in K_{1}\left(\mu_{1}-\mu_{2}\right)+\left(r \in \frac{K_{1}}{K_{2}}\right) K_{2}\left(\mu_{1}-\mu_{2}\right)=0 \\
& \therefore \sum_{i=1}^{N} w_{i}^{B}=\sum_{i=1}^{N} w_{i}^{A} .
\end{aligned}
$$

\section{Part II}

Show $\sum_{i=1}^{k} w_{i}^{B} \geq \sum_{i=1}^{k} w_{i}^{A}, k \leq N$, with at least one strict inequality when $w_{2}^{1}<w_{2}^{2} \leq$ $w_{1}^{1}<w_{1}^{2}$. The $w_{i}$ are ordered so that $w_{i} \leq w_{j}$ for $i<j$. Therefore, after categorization:

the first $p_{1} n_{11}+p_{2} n_{21}=s_{1}^{A}$ individuals receive $w_{2}^{1}$

the next $p_{1} n_{12}+p_{2} n_{22}=s_{2}^{A}$ individuals receive $w_{2}^{2}$

the next $\left(1-\mathrm{p}_{1}\right) \mathrm{n}_{11}+\left(1-\mathrm{p}_{2}\right) \mathrm{n}_{21}=\mathrm{s}_{3}^{\mathrm{A}}$ individuals receive $\mathrm{w}_{1}^{1}$

the next $\left(1-p_{1}\right) n_{12}+\left(1-p_{2}\right) n_{22}=s_{4}^{A}$ individuals receive $w_{1}^{2}$

Call this distribution "the A distribution".

Before categorization, since $W_{2}<W_{1}$,

the first $p_{1}\left(n_{11}+n_{12}\right)+p_{2}\left(n_{21}+n_{22}\right)=s_{1}^{B}=s_{1}^{\Lambda}+s_{2}^{\Lambda}$ individuals receive $w_{2}$

the next $\left(1-p_{1}\right)\left(n_{11}+n_{12}\right)+\left(1-p_{2}\right)\left(n_{21}+n_{22}\right)=s_{2}^{B}=s_{3}^{A}+s_{4}^{A}$ individuals receive $w_{1}$

Call this distribution "the B distribution". Since $W_{2}^{1}<W_{2}$ it follows that the first $s_{1}^{A}(s)$ individuals after categorization receive less total wealth than do the first $s_{1}^{A}(s)$ individuals before categorization so that $\sum_{i=1}^{k} w_{i}^{A}<\underset{i=1}{s_{i}} w_{i}^{B}$, $k \leq s_{1}^{A}$. The proof proceeds by calculating the difference $\underset{i=1}{s_{1}^{A}{ }_{i}^{A} w_{i}^{B}}-$ $\sum_{i=1}^{s_{1}^{A} w_{i}^{A}}$ and then showing that $\sum_{i=1}^{k} w_{i}^{A}<\sum_{i=1}^{k} w_{i}^{B}$ for $k<N$. Since $\sum_{i=1}^{N} w_{i}^{A}=\sum_{i=1}^{N} w_{i}^{B}$ this result implies that as more individuals are accounted for total wealth for the A distribution (individuals after categorization) never catches up to total 
wealth in the B distribution (individuals before catcgorization) until the $\mathrm{N}^{\text {th }}$ individual is considered.

(i)

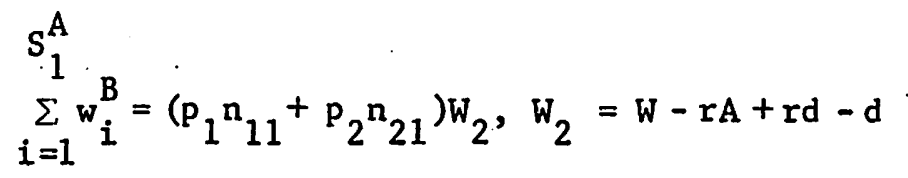

$$
s_{1}^{A} \cdot w_{i}^{A}=\left(p_{1} n_{11}+p_{2} n_{21}\right) w_{2}^{1}, w_{2}^{1}=w-r A_{1}+r d-d
$$

$$
\begin{aligned}
& i=1 \\
& \Rightarrow \sum_{i=1}^{s_{1}^{A} w_{i}^{B}-} \sum_{i=1}^{s_{1}^{A} w_{i}^{A}}=\left(p_{1} n_{11}+p_{2} n_{21}\right) r\left(A_{1}-A\right) \\
& =\left(p_{1} n_{11}+p_{2} n_{21}\right) r \in\left(\mu_{1}-\mu_{2}\right)
\end{aligned}
$$

(ii) Consider the next group of $s_{2}^{A}$ individuals, remembering that $s_{1}^{A}+s_{2}^{A}=s_{1}^{B}$.

$$
\begin{aligned}
& \sum_{i=s_{1}^{A}}^{s_{1}^{B}} w_{i}^{B}=\left(p_{1} n_{12}+p_{2} n_{22}\right) w_{2} \\
& s_{1}^{B} \\
& \sum_{i=s_{1}^{A}}^{A} w_{i}^{A}=\left(p_{1} n_{12}+p_{2} n_{22}\right) w_{2}^{2}, w_{2}^{2}=w-r A_{2}+r d-d
\end{aligned}
$$

Since $W_{2}^{2}>W_{2}$, total wealth for the A distribution increases more quickly than for the B distribution. However, as the following demonstrates, the total wealth for the A distribution never "catches up" to the B distribution.

$$
\begin{aligned}
& \sum_{1}^{B} \quad w_{i}^{A}-\ddot{Z}_{i=S_{1}^{A}}^{B} \quad w_{i}^{B}=\left(P_{1} n_{12}+p_{2} n_{22}\right) r\left(A-A_{2}\right) \\
& i=S_{1}^{A}+1 \quad i=s_{1}^{A}+1=\left(p_{1} n_{12}+p_{2} n_{22}\right) r \in k\left(\mu_{1}-\mu_{2}\right)>0
\end{aligned}
$$

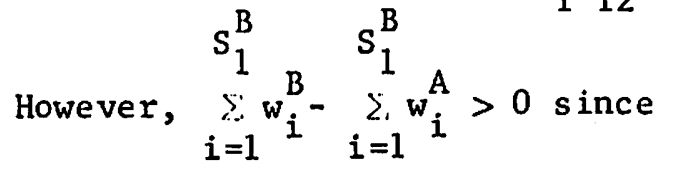

$$
\begin{aligned}
& s_{1}^{B} s_{i=1}^{B} s_{1}^{B}-\sum_{i=1}^{A} w_{i}^{A}=\left(p_{1} n_{11}+p_{2} n_{21}\right) r \epsilon\left(\mu_{1}-\mu_{2}\right) \\
& \quad-\left(p_{1} n_{12}+p_{2} n_{22}\right) r \epsilon k\left(\mu_{1}-\mu_{2}\right) \\
& =r \epsilon\left(\mu_{1}-\mu_{2}\right)\left[\left(p_{1} n_{11}+p_{2} n_{21}\right)-k\left(p_{1} n_{12}+p_{2} n_{22}\right)\right]
\end{aligned}
$$


This expression is positive provided the term in square brackets is positive. Now $\left(p_{1} n_{11}+p_{2} n_{21}\right)-\frac{k_{1}}{k_{2}}\left(p_{1} n_{12}+p_{2} n_{22}\right)>0$

$\Leftrightarrow p_{1} \frac{n_{11}}{k_{1}}+p_{2} \frac{n_{21}}{k_{1}}-p_{1} \frac{n_{12}}{k_{2}}-p_{2} \frac{n_{22}}{k_{2}}>0$ upon dividing by $k_{1}$

$\Leftrightarrow p_{1}\left(q_{11}-q_{12}\right)+p_{2}\left(q_{21}-q_{22}\right)>0$

$\therefore \quad \Rightarrow P_{1}(\epsilon+\epsilon k)+P_{2}(-\epsilon-\epsilon k)>0$

$\Leftrightarrow \quad\left(p_{1}-p_{2}\right)(\epsilon+\epsilon k)>0$

which holds since $p_{1}>p_{2}$. Therefore, after the $s_{1}^{B}$ individuals are considered it is still true that $\sum_{i=1}^{s_{1}^{B}} w_{i}^{B}>\sum_{i=1}^{s_{1}^{B}} w_{i}^{A}$

(iii) Consider the next group of $\mathrm{s}_{3}^{\mathrm{A}}$ individuals.

$$
\begin{aligned}
& \sum_{1}^{s^{B}+s_{3}^{A}} w_{i}^{B}=\left[\left(1-p_{1}\right) n_{11}+\left(1-p_{2}\right) n_{21}\right] w_{1}, w_{1}=w-r A \\
& i=s_{1}^{B}+1 \\
& s_{1}^{B}+s_{3}^{A} \\
& \sum_{i=w_{1}^{B}+1}^{A} w_{i}^{A}=\left[\left(1-p_{1}\right) n_{11}+\left(1-p_{2}\right) n_{21}\right] w_{1}^{1}, w_{1}^{1}=w-r A_{1}
\end{aligned}
$$

Since $W_{1}>W_{1}^{1}$, total wealth for the $B$ distribution increases more quickly than it does for the $A$ distribution. Therefore, $\sum_{i=1}^{k} w_{i}^{B}>\sum_{i=1}^{k} w_{i}^{A}, k \leq s_{1}^{A}+s_{2}^{A}+s_{3}^{A}$.

(iv) Consider the next group of $\mathrm{s}_{4}^{\mathrm{A}}$ individuals. For this group the individuals in the $A$ distribution receive wealth $W_{1}^{2}$ which is greater than that for those in the $B$ distribution, who receive wealth $w_{1}$ (i.e., $w_{1}^{2}>w_{1}$ ). However, although $\sum_{i=1}^{k} w_{i}^{A}$ increases more rapidly than does $\sum_{i=1}^{k} w_{i}^{B}$, when the final group of individuals $i=1$ is being considered, it is not until the $N^{\text {th }}$ individual is included that $\sum w_{i}^{A}$ "catches up" to $\sum w_{i}^{B}$. That is, if $\sum_{i=1}^{k} w_{i}^{A} \geq \sum_{i=1}^{k} w_{i}^{B}$ for $s_{1}^{A}+s_{2}^{A}+s_{3}^{A} \leq k<N$ then 
it would not be possible that $\sum_{i=1}^{N} w_{i}^{A}: \sum_{i=1}^{N} w_{i}^{B}$ (which was shown to be true in Part I of the proof).

\section{Part III}

Show $\sum_{i=1}^{k} w_{i}^{B} \geq \sum_{i=1}^{k} w_{i}^{A}, k \leq N$, with at least one strict inequality when $w_{2}^{1}<w_{1}^{1} \leq w_{2}^{2}<$ $w_{1}^{2}$. The $w_{i}$ are ordered so that $w_{i} \leq w_{j}$ for $i<j$. Therefore, after categorization: the first $p_{1} n_{11}+p_{2} n_{21}=s_{1}^{A}$ individuals receive $w_{2}^{1}$ the next $\left(1-\mathrm{p}_{1}\right) \mathrm{n}_{11}+\left(1-\mathrm{p}_{2}\right) \mathrm{n}_{21}=\mathrm{s}_{2}^{\mathrm{A}}$ individuals receive $\mathrm{w}_{1}^{1}$ the next $p_{1} n_{12}+p_{2} n_{22}=s_{3}^{A}$ individuals receive $w_{2}^{2}$ the next $\left(1-\mathrm{p}_{1}\right) \mathrm{n}_{12}+\left(1-\mathrm{p}_{2}\right) \mathrm{n}_{22}=\mathrm{s}_{4}^{\mathrm{A}}$ individuals receive $\mathrm{w}_{1}^{2}$

Once again, call this "the A distribution". Also, before categorization, the first $p_{1}\left(n_{11}+n_{12}\right)+p_{2}\left(n_{21}+n_{22}\right)=s_{1}^{B}$ individuals receive $w_{2}$ the next $\left(l-p_{1}\right)\left(n_{11}+n_{12}\right)+\left(l-p_{2}\right)\left(n_{21}+n_{22}\right)=s_{2}^{B}$ individuals receive $w_{1}$ Call this "the B distribution".

In this part of the proof the algebra from Part II is used to show that the Lorenz curve for the B distribution (representing the distribution of wealth before categorization) dominates the Lorenz curve for the A distribution (representing the distribution of wealth after categorization).

There are two possible cases. Although $s_{1}^{A}<s_{1}^{B}$ and $s_{4}^{A}<s_{2}^{B}$ it may be the case that either

(a) $s_{1}^{A}+s_{2}^{A}>s_{1}^{B}$, or
(b) $s_{1}^{A}+s_{2}^{A}<s_{1}^{B}$

Consider case (a) first.

For the first $S_{1}^{A}$ individuals the Lorenz curve for $B$ rises more rapidly than for $A$ (see ( $i$ ) of Part II of this proof). For the next $s_{1}^{B}-s_{1}^{A}$ individuals the members of the $A$ distribution receive wealth $W_{1}^{1}$ while those in the $B$ 
distribution receive $W_{2}$. Even if members of the A distribution received wealth $\mathrm{W}_{2}^{2}$ ( $z \mathrm{~W}_{1}^{1}$ by assumption), Part II, (ii) of this proof shows that the Lorenz curve for A would still be below that for $B$ (i.e., $\sum_{i=1}^{k} w_{i}^{A}<\sum_{i=1}^{k} w_{i}^{B}$ for $k \leq s_{1}^{B}$ ). Therefore, a fortiori, the Lorenz curve for $A$ will be below that for $B$ after $s_{1}^{B}$ individuals have been considered.

Now, the remainder of the $\mathrm{S}_{2}^{\mathrm{A}}$ individuals from the A distribution receive wealth $w_{1}^{1}$ while those in the $B$ distribution receive $w_{1}>w_{1}^{1}$. Therefore, the Lorenz curve for the A distribution falls even further below the Lorenz curve for $B$ (i.e., $\sum_{i=1}^{k} w_{i}^{A}<\sum_{i=1}^{k} w_{i}^{B}$ for $k \leq s_{1}^{A}+s_{2}^{A}$ ). For the third group the $s_{3}^{A}$ individuals from $A$ receive wealth $w_{2}^{2}$ while those in $B$ receive $W_{1}$. Although $w_{2}^{2}$ may be greater than $w_{1}$ the Lorenz curve for A cannot intersect (i.e., "catch up" to) that for $B$. This result must hold since for the last group the Lorenz curve for A rises more quickly than that for $B\left(W_{1}^{2}>W_{1}\right)$, and if the Lorenz curve for $A$ had intersected the $B$ curve when considering the third group then the condition that $\sum_{i=1}^{N} w_{i}^{A}=\sum_{i=1}^{N} w_{i}^{B}$ holds (a result proved in Part I) would be contradicted. Therefore, the Lorenz curve for the B distribution once again dominates that for the A distribution. This result is illustrated in Figure III.

Now consider case (b) with $s_{1}^{A}+s_{2}^{A}<s_{1}^{B}$. Upon consideration of the first $s_{1}^{A}$ individuals, $\sum_{i=1}^{k} w_{i}^{A}<\sum_{i=1}^{k} w_{i}^{B}$ for $0<k \leq s_{1}^{A}$, since $w_{2}^{1}<w_{2}$. Since $w_{1}^{1} \leq w_{2}^{2}$ it follows from part II, (ii) that $\sum_{i=1}^{k} w_{i}^{A}<\sum_{i=1}^{k} w_{i}^{B}$ for $k \leq s_{1}^{A}+s_{2}^{A}$ (this result is the same as for case (a)). Upon considering the next $s_{1}^{B}-s_{1}^{A}+s_{2}^{A}$ individuals (from $S_{3}^{A}$ for the $A$ distribution and $S_{1}^{B}$ from the $B$ distribution) it also follows from Part II, (ii) that

$$
\sum_{i=1}^{k} w_{i}^{A}<\sum_{i=1}^{k} w_{i}^{B} \text { for } 0<k \leq s_{1}^{B}
$$


After $s_{1}^{B}$ individuals have been considered (see point $J$ in Figure IV below) the Lorenz curve for the A distribution will not have intersected ("caught up to") the Lorenz curve for the B distribution. Therefore, since the remaining segments of the A curve become increasingly more steep and $\sum_{i=1}^{N} w_{i}^{A}=\sum_{i=1}^{N} w_{i}^{B}$ it follows that the Lorenz curve of the A distribution is dominated by that of the $B$ curve. This result is illustrated by Figure IV. 